\title{
Eine optimale Lösung? Eine Replik auf Gerd Strohmeier in Heft 3/2007 der ZParl ${ }^{*}$
}

\author{
Harald Schoen
}

Gerd Strohmeier plädiert in Heft 3/2007 der Zeitschrift für Parlamentsfragen für eine Reform des Wahlsystems zum Deutschen Bundestag. Auf der Grundlage einer ausführlichen Diagnose von Defiziten des personalisierten Verhältniswahlsystems schlägt er vor, eine Mehrheitswahl mit proportionaler Zusatzliste einzuführen. Dieses segmentierte Wahlsystem sieht für jeden Wähler wie bisher zwei Stimmen vor. Bei der Stimmverrechnung sollen drei Viertel, besser aber fünf Sechstel der Mandate in (verkleinerten) Einerwahlkreisen nach dem Prinzip der relativen Erststimmenmehrheit verteilt werden, der Rest nach dem Zweitstimmenanteil, wobei weder eine Sperrklausel noch eine Verrechnung mit den Direktmandaten vorgesehen ist. ${ }^{1}$ Obgleich als „exemplarische Diskussionsgrundlage "2 deklariert, gilt diese Verrechnungsmethode dem Autor als „optimale Lösung der mit der Wahlsystemfrage aufgeworfenen Regierbarkeits- und Gerechtigkeitsproblematik“, weshalb er an die Parteien der Großen Koalition appelliert, diese Variante der abgeschwächten Mehrheitswahl einzuführen. ${ }^{3}$

Strohmeiers facettenreiche Argumentation bietet zahlreiche Ansatzpunkte zur Diskussion, und zwar sowohl hinsichtlich der Diagnose von Mängeln des gegenwärtigen Wahlsystems als auch hinsichtlich seines Therapievorschlags. Der vorliegende Beitrag konzentriert sich im Wesentlichen darauf, den Reformvorschlag auf seine Tauglichkeit hin zu überprüfen, die vom Autor gesetzten Ziele zu erreichen. ${ }^{4}$ Als „primäres Repräsentationsziel [gilt ihm, H.S.] eine absolute Parlamentsmehrheit für eine Partei und als sekundäres eine gewisse [...] parlamentarische Repräsentation der (wichtigsten) kleinen Parteien "5.

Zweifelsohne bietet die gemäßigte Mehrheitswahl kleinen Parteien eine Chance, im Bundestag vertreten zu sein. Allerdings lohnt sich ein genauerer Blick darauf, wie viele Abgeordnete diese kleinen Parteien stellen würden. Gehen wir von einem Viertel Listenmandaten und zehn Prozent Zweitstimmen für eine Partei aus, so ergeben sich in einem Parlament mit 598 Sitzen 15 Mandate; sinkt der Anteil der Zweitstimmensitze auf das von Strohmeier favorisierte Sechstel, werden zehn Mandate erzielt. Das heißt, Parteien wie die FDP würden im Bundestag nicht den Fraktionsstatus erreichen; dazu wären in der ersten

Gerd Strohmeier, Ein Plädoyer für die „gemäßigte Mehrheitswahl“: optimale Lösung für Deutschland, Vorbild für Österreich und andere Demokratien, in: ZParl, 38. Jg. (2007), H. 3, S. 578 -590 .

1 Vgl. ebenda, S. 587 f.

2 Ebenda, S. 587.

3 Ebenda, S. 590.

4 Beschränkte man sich nicht auf eine immanente Betrachtung, könnte man beispielsweise fragen, ob die Mehrheitsverhältnisse und Koalitionsmöglichkeiten des Jahres 2005 auf absehbare Zeit als fixiert zu betrachten sind und wir daher unausweichlich vor der Alternative: Große Koalition oder Regierbarkeitskrise stehen (S. 580 f.). Ebenso ist nicht ohne weiteres zu erkennen, warum es problematisch sein soll, wenn eine vor der Wahl angekündigte Koalition nach der Wahl gegen die stimmen- und mandatstärkste Partei geschlossen wird und die Regierung übernimmt (S. 584).

5 Ebenda, S. 585. 
Konstellation 20 Prozent, in der zweiten 30 Prozent der Zweitstimmen erforderlich. Unter den momentanen politischen Kräfteverhältnissen würde daher der Bundestag wohl aus zwei Fraktionen, nämlich CDU/CSU und SPD, und einigen Gruppen bestehen. Auch wenn ihre Rechte in den letzten Jahren gestärkt worden sind, sind die Gruppen den Fraktionen nicht gleichgestellt, so dass im Bundestag gewissermaßen eine Zweiklassengesellschaft entstünde. Selbst wenn die Gruppen den Fraktionen rechtlich gleichgestellt würden, ergäben sich aus der kleinen Zahl von Abgeordneten in einer Gruppe praktische Probleme. Denn zehn oder 15 Parlamentarier dürften es nicht leicht haben, in allen Gremien des Bundestages wettbewerbsfähig mitzuwirken und den Fraktionen Paroli zu bieten. Im Ergebnis könnte man darin durchaus eine Tendenz zur „massiven parlamentarischen Zurückdrängung“ der kleinen Parteien sehen, die Strohmeier „,höchst schädlich für die Politische Kultur und damit auch die Stabilität des gesamten politischen Systems "6 hält.

Vor dem Hintergrund dieser absehbaren Konsequenzen des gemäßigten Mehrheitswahlsystems für die Zusammensetzung des Bundestages stellt sich die Frage, ob dieses Wahlverfahren politisch durchsetzbar ist. Die Öffentlichkeit könnte in seiner Einführung einen Versuch der die Große Koalition tragenden Parteien sehen, ihre parlamentarische Übermacht für eigennützige Zwecke einzusetzen. Paradoxerweise könnte daher das Plädoyer für die gemäßigte Mehrheitswahl von manchem Beobachter als ein Appell an die Große Koalition zu jenem Machtmissbrauch gelesen werden, dem der Autor mit dieser Wahlsystemreform gerade vorbeugen will.7 Selbst wenn man diese Überlegung ausblendet, ist nicht sicher, wie eine Gesellschaft, die seit Jahrzehnten Parlamente (primär) nach dem Verhältniswahlprinzip gewählt hat, auf ein System reagieren würde, dessen erklärtes Ziel es ist, einer Partei(enformation) mit gut einem Drittel der Zweitstimmen, nämlich Union oder SPD, die absolute Mehrheit der Mandate zu verschaffen. Ein solches Wahlsystem könnte den Repräsentations- und Gerechtigkeitsvorstellungen eines erheblichen Teils des Elektorats widersprechen und daher auf Widerstand stoßen.

Es ist allerdings nicht auszuschließen, dass sich Abgeordnete und Bürger von der überragenden Bedeutung von Einparteiregierungen überzeugen lassen, daher andere Überlegungen hintanstellen und die gemäßigte Mehrheitswahl ausschließlich nach deren Fähigkeit beurteilen, für parteipolitisch „einfarbige“ Regierungen zu sorgen. Um zu prüfen, wie die proportional ergänzte Mehrheitswahl in dieser Hinsicht abschneidet, bietet es sich an, dieses Wahlsystem auf das Ergebnis der Bundestagswahl 2005 anzuwenden, das den Ausgangspunkt für den diskutierten Vorschlag darstellt. Dabei wird von 299 Direktmandaten und 60 Sitzen - also einem Sechstel aller Mandate - ausgegangen, die gemäß dem Zweitstimmenergebnis proportional ohne gesetzliche Sperrklausel verteilt werden. Es resultiert ein Parlament mit 359 Sitzen, von denen 166 auf die SPD entfallen, 172 auf CDU und CSU, acht auf die Linkspartei, je sechs auf FDP und Bündnis 90/Die Grünen sowie einer auf die NPD. Offensichtlich erreicht keine Partei die absolute, für die Kanzlerwahl benötigte Mehrheit von 180 Stimmen. Dem vorgeschlagenen Wahlsystem gelingt es also nicht, eine Einparteiregierung hervorzubringen; dazu müssten Union oder SPD bei den Direktoder Zweitstimmenmandaten deutlich besser als 2005 abschneiden. Anders als das personalisierte Verhältniswahlsystem ermöglicht die proportional ergänzte Mehrheitswahl jedoch eine kleine Koalition, nämlich ein Bündnis aus CDU/CSU und der Linkspartei. Da diese

6 Ebenda.

7 Vgl. ebenda, S. 582. 
Koalition jedoch politisch kaum möglich ist, bleiben als realistischere Optionen eine Große Koalition oder aber ein Dreierbündnis. Dieser Kernbefund gilt auch dann, wenn man von einem Parlament mit 598 Mandaten ausgeht oder wenn nicht ein Sechstel, sondern ein Viertel der Mandate gemäß den Zweitstimmenanteilen der Parteien verteilt werden. ${ }^{8}$ Die gemäßigte Mehrheitswahl lässt somit die Möglichkeiten der Regierungsbildung praktisch unverändert. Das Ziel einer Einparteiregierung würde allenfalls dann erreicht, wenn man keine oder sehr wenige Mandate gemäß dem Zweitstimmenanteil verteilte, also praktisch ein reines Mehrheitswahlsystem einführte, das Strohmeier jedoch verworfen hat. ${ }^{9}$

Nun könnte man einwenden, in den bisher angestellten Überlegungen sei übersehen worden, dass Wahlsysteme nicht nur mechanische, sondern auch psychologische Effekte zeitigten, weshalb man die Stimmenverteilung aus dem Jahr 2005 nicht ohne weiteres einer Wirkungsanalyse zugrunde legen dürfe. Psychologische Effekte sind naturgemäß weniger leicht kalkulierbar als mechanische, doch soll dennoch versucht werden, sie zu berücksichtigen. Inwieweit Bürger unter einem proportional ergänzten Mehrheitswahlsystem Anreizen zu taktischem Stimmverhalten folgten, lässt sich nicht mit Sicherheit sagen. Doch spricht die Erfahrung mit der personalisierten Verhältniswahl dafür, darin ein eher marginales Phänomen zu sehen. ${ }^{10}$ Soweit Bürger bei der Wahl taktischen Anreizen folgen, ist zudem nicht ausgemacht, dass dies notwendig die mehrheitsbildende Wirkung des Wahlsystems begünstigte. Taktisch vergebene Erststimmen können die Mandatsstärke der beiden potentiellen alleinigen Regierungsparteien kaum weiter steigern. Mit der Zweitstimme könnten einige Bürger, die die Regierungsbildung beeinflussen wollen, durchaus gegen ihre politische Präferenz für Union oder SPD votieren. Zugleich ist jedoch zu bedenken, dass die gemäßigte Mehrheitswahl im Hinblick auf die Zweitstimme im Vergleich zur bestehenden Fünfprozenthürde die Repräsentationsschwelle senkt, da beispielsweise bei 100 proportional ohne Sperrklausel vergebenen Mandaten für den Parlamentseinzug ein Prozent der Zweitstimmen genügt. Es könnten also einige Bürger aufrichtig für eine kleine Partei stimmen, die bislang für eine große Partei votierten, um ihre Stimme nicht zu verschenken. Darüber hinaus könnten einige Bürger ganz bewusst gegen ihre Präferenz kleine Parteien wählen, um etwa eine absolute Mehrheit einer Partei zu verhindern. Im Hinblick auf die Parteieliten ist nicht erkennbar, dass die neue Anreizkonstellation kleine Parteien unmittelbar zum Rück-

8 Bei der Betrachtung eines Parlaments mit 598 Mandaten wurde bei der Verteilung der mehr als 299 Direktmandate von den Direktmandatsanteilen bei der Wahl 2005 ausgegangen. Dieses Szenario setzt die Chancen auf eine absolute Mandatsmehrheit von Union oder SPD eher zu hoch als zu niedrig an, da kleinere Wahlkreise es anderen Parteien als CDU, CSU und SPD erleichtern könnten, Direktmandate zu erringen.

9 Vgl. Gerd Strohmeier, a.a.O., S. 585. Selbst in diesem Fall wäre jedoch nicht garantiert, dass die Partei mit den meisten Stimmen die meisten Sitze erhält und die Regierung stellt, da in einem Wahlsystem mit mehreren Wahlkreisen die Sitzzahl einer Partei nicht nur von ihrer Gesamtstimmenzahl, sondern auch von deren regionaler Verteilung abhängt. Siehe etwa Ron J. Johnston I Charles J. Pattie / Daniel Dorling / David J. Rossiter / Helen Tunstall / Ian McAllister, New Labour Landslide - Same Old Electoral Geography, in: British Elections and Parties Review, 8. Jg. (1998), S. $35-64$.

10 Vgl. Bernhard Weßels, „Aufrichtiges“ Wahlverhalten: Parteiorientierungen, Kandidatenbeurteilungen und generalisierte Politikdistanzen, in: Hans-Dieter Klingemann / Max Kaase (Hrsg.), Wahlen und Wähler. Analysen aus Anlass der Bundestagswahl 1998, Wiesbaden 2001, S. 695 -716 . 
zug oder zur Fusion mit Union oder SPD bewegen würde. ${ }^{11}$ Auch unter Berücksichtigung psychologischer Wahlsystemeffekte erscheint es somit zweifelhaft, ob die gemäßigte Mehrheitswahl Einparteiregierungen herbeiführen kann. ${ }^{12}$

Als Fazit bleibt festzuhalten, dass das proportional ergänzte Mehrheitswahlsystem kleine Parteien im Bundestag empfindlich zugunsten großer Parteien schwächt. Zugleich ist es nicht in der Lage, in der Bundesrepublik Deutschland unter den gegebenen Bedingungen eine Parlamentsmehrheit für eine Partei herbeizuführen - und das dürfte es unter Strohmeiers Annahmen ${ }^{13}$ auch künftig nicht leisten können. Selbst wenn man die Prämissen ihres Verfechters zugrunde legt, vermag die gemäßigte Mehrheitswahl somit nicht zu überzeugen. Daher sind ihre Chancen, die personalisierte Verhältniswahl in absehbarer Zeit als Bundestagswahlsystem abzulösen, als gering einzuschätzen.

11 Dabei ist unter anderem zu bedenken, dass mit der Einführung der gemäßigten Mehrheitswahl auf Bundesebene dieses System nicht automatisch auch auf Landesebene eingeführt würde.

12 Analog ändert die Berücksichtigung psychologischer Effekte das Ergebnis zu den kleinen Parteien nicht wesentlich.

13 Vgl. Gerd Strohmeier, a.a.O., S. 581.

\title{
Mit dem Internet in die parlamentarische Provinzialität
}

\author{
Erich Röper
}

Als Voraussetzung demokratischer Willensbildung fordern das Grundgesetz und die Landesverfassungen die Öffentlichkeit der Parlamentssitzungen. Es gilt folgerichtig für alle parlamentarischen Vorgänge, jedenfalls grundsätzlich, da etwa die Sitzungen der Ausschüsse meist nichtöffentlich sind. Auch die den Plenardebatten zugrunde liegenden Drucksachen müssen allgemein zugänglich sein, den Abgeordneten aller Parlamente, ihren Fraktionen und Mitarbeitern sowie Interessierten. Obwohl sie in allen Parlamenten, Fraktionen und vielen Bibliotheken vorlagen, erreichten sie in Papierform nur einen beschränkten Personenkreis. Daher gilt das Internet als Königsweg.

\section{Parlamentarische Dokumente in Papierform: Chance auf umfassende politische Kenntnis}

Die Möglichkeit für Abgeordnete und Fraktionen, sich einen Überblick über das parlamentarische und politische Geschehen in Bund und Ländern zu verschaffen, wurde seit einiger Zeit eingeschränkt: Seit dem Jahr 2002 stellten der Bundestag und die Landtage den Drucksachenaustausch nach und nach ein, seit 2005 auch ihren Versand an die meisten Parlamentsbibliotheken. Der Austausch wurde auf das Internet konzentriert; Papierexemplare erhalten nur noch der Bundestag, der Bundesrat und der Landtag von Niedersachsen. 